\title{
Chronic fatigue syndrome: an emerging sequela in COVID-19 survivors?
}

\author{
Elisa Mantovani ${ }^{1}\left[\right.$ (D) Sara Mariotto ${ }^{1}$. Daniele Gabbiani ${ }^{2} \cdot$ Gianluigi Dorelli $^{2} \cdot$ Silvia Bozzetti $^{1} \cdot$ Angela Federico $^{1}$. \\ Serena Zanzoni ${ }^{3} \cdot$ Domenico Girelli $^{2}$ - Ernesto Crisafulli ${ }^{2} \cdot$ Sergio Ferrari ${ }^{1} \cdot$ Stefano Tamburin $^{1}$ (I)
}

Received: 11 April 2021 / Revised: 6 July 2021 / Accepted: 13 July 2021 / Published online: 2 August 2021

(c) Journal of NeuroVirology, Inc. 2021

\begin{abstract}
SARS-CoV-2 survivors may report persistent symptoms that resemble myalgic encephalomyelitis/chronic fatigue syndrome (ME/CFS). We explored (a) ME/CFS-like symptom prevalence and (b) whether axonal, inflammatory, and/or lung changes may contribute to ME/CFS-like symptoms in SARS-CoV-2 survivors through clinical, neuropsychiatric, neuropsychological, lung function assessment, and serum neurofilament light chain, an axonal damage biomarker. ME/CFS-like features were found in $27 \%$ of our sample. ME/CFS-like group showed worse sleep quality, fatigue, pain, depressive symptoms, subjective cognitive complaints, Borg baseline dyspnea of the 6-min walking test vs. those without ME/CFS-like symptoms. These preliminary findings raise concern on a possible future ME/CFS-like pandemic in SARS-CoV-2 survivors.
\end{abstract}

Keywords Cognition · Mood alterations · COVID-19 · Fatigue $\cdot$ Functional neurological disorders $\cdot$ Viral infection

\section{Introduction}

COVID-19 long-haulers fail to revert to normal routine after SARS-CoV-2 infection and report persistent debilitating symptoms, i.e., fatigue, "brain fog", pain, disrupted sleep, and mood alterations (Rubin 2020).

Chronic COVID-19 may resemble myalgic encephalomyelitis/chronic fatigue syndrome (ME/CFS), a well-documented post-viral condition (Rubin 2020) affecting $0.5-2.5 \%$ of the population (Johnston et al. 2013). $\mathrm{ME} / \mathrm{CFS}$ is characterized by severe post-exertional fatigue not improving with rest, subjective/objective cognitive difficulties, pain, sleep abnormalities, neurosensory, immune, gastro-intestinal disturbances, and cellular energy

Elisa Mantovani

elisa.mantovani@univr.it

Stefano Tamburin

stefano.tamburin@univr.it

1 Department of Neurosciences, Biomedicine and Movement Sciences, Neurology Section, University of Verona, Verona, Italy

2 Department of Medicine, Internal Medicine Section D, University of Verona, Verona, Italy

3 Centro Piattaforme Tecnologiche, University of Verona, Verona, Italy metabolism dysfunction (Carruthers et al. 2011). Several ME/CFS inconclusive pathophysiological hypotheses have been proposed, including immunological changes (Carruthers et al. 2011). ME/CFS cognitive complaints have been suggested to share pathogenetic commonalities with functional neurological disorders (Teodoro et al. 2018).

Studies in survivors of other coronavirus infections, namely, Severe Acute Respiratory Syndrome (SARS) and Middle East respiratory syndrome, documented a ME/CFSlike picture (Lam et al. 2009; Lee et al. 2019), supporting a link with viral infection (Ahmed et al. 2020), but data on SARS-CoV-2 are preliminary (Townsend et al. 2020).

Serum levels of neurofilament light chain (NfL), a biomarker of axonal damage, may be increased during COVID19 acute phase (Aamodt et al. 2021), even in the absence of overt neurological signs, suggesting a possible SARS-CoV-2 neuronal trophism (Mariotto et al. 2020). The possible association between chronic fatigue and serum NfL levels has not been determined in this condition, yet.

Persistent lung impairment has been reported after SARSCoV-2 infection and might contribute to chronic COVID-19 (Rubin 2020).

Our aims were to explore (a) the extent of ME/CFS-like symptoms and (b) whether baseline inflammatory markers, and axonal, and/or lung abnormalities may contribute to ME/CFS-like symptoms in COVID-19 survivors. To 
this aim, a group of COVID-19 survivors underwent a multidimensional evaluation, including neuropsychiatric, neuropsychological, lung function assessment, and $\mathrm{NfL}$ serum level measurement.

\section{Methods}

\section{Subjects}

Inpatients and outpatients positive to SARS-CoV-2 PCR testing in February-May 2020 were recruited at the Department of Internal Medicine, Verona University Hospital, Italy, after informed consent.

Inclusion criteria include (a) age 18-65 years; (b) no history of neurological, cerebrovascular, psychiatric disorders, or substance use disorders that might interfere with cognition; (c) > 6-month follow-up after SARS-CoV-2 infection; (d) negative nasopharyngeal swab test; and (e) no history of fatigue before SARS-CoV-2 infection.

Socio-demographic data, baseline comorbidities, and clinical and inflammatory features of SARS-CoV-2 infection features were collected. The study was approved by the local ethic committee (\#2785CESC).

\section{ME/CFS-like symptoms}

ME/CFS-like symptoms were assessed according to the ME International Consensus Criteria (Carruthers et al. 2011) with a semi-structured clinical interview. Patients were classified as typical ME/CFS-like if they met (a) post-exertional neuroimmune exhaustion criteria, (b) $\geq 1$ neurological impairment symptom, (c) $\geq 1$ immune/ gastro-intestinal/genitourinary impairment symptom, and (d) $\geq 1$ energy metabolism/transport impairment symptom. Patients meeting post-exertional neuroimmune exhaustion criteria but $1-2$ of the remaining criteria (points $b-d$ ) were classified as atypical ME/CFS-like. Typical and atypical ME/CFS-like patients were lumped in the ME/ $\mathrm{CFS}+$ group, while other patients represented the ME/ $\mathrm{CFS}$ - group.

\section{Neuropsychiatric symptoms}

Fatigue was explored with the Multidimensional Fatigue Inventory (MFI). The Pittsburgh Sleep Quality Index (PSQI) evaluated sleep quality over the previous month. The Hospital Anxiety and Depression Scale (HADS) assessed mood changes. Pain severity was evaluated with a $0-10$ Numerical Rating Scale.

\section{Neuropsychological assessment}

Subjective cognitive complaints (e.g., attention, concentration, and memory difficulties) were assessed through patients' reports.

Global cognition was explored with the Montreal Cognitive Assessment (MoCA). A reduced neuropsychological test battery was administered to assess the most frequently affected cognitive domains in ME/CFS. Attention and psychomotor speed were evaluated with the Symbol Digit Test and executive function was explored with the Stroop test.

\section{Neurofilament light chain}

Investigators blinded to clinical data measured serum NfL levels in duplicated using SIMOA Nf-light kit in SR-X immunoassay analyser, Simoa (Quanterix Corporation, Billerica, Massachusetts, USA), which runs ultrasensitive paramagnetic bead-based enzyme-linked immunosorbent assays (Mariotto et al. 2019). Age-matched healthy controls and normative NfL values were used as comparison (Hviid et al. 2020).

\section{Lung function}

Lung function was performed according to the International Recommendations (Miller et al. 2005). A flow-sensing spirometer (Jaeger MasterScreen PFT System) was used. Forced vital capacity (FVC), forced expiratory volume in the first second (FEV1), and total lung capacity (TLC) were recorded. FEV1/ FVC ratio was taken as index of airflow obstruction. Carbon monoxide diffusion capacity (DLCO) and carbon monoxide transfer coefficient (KCO) were measured by single breath method. FEV1, FVC, TLC, DLCO, and KCO were expressed as percentage of the predicted values. The arterial blood partial pressure of carbon dioxide and oxygen was measured. Walking capacity was assessed by 6 -min walking test (6MWT) according to the reference equation for healthy adults, and the individual's perceived dyspnea and fatigue at baseline and at end-effort were measured with a 10-point modified Borg scale.

\section{Statistical analysis}

Continuous variables were assessed with non-parametric Mann-Whitney U test. Chi square test was applied to categorical variables. Statistical significance was set at $p<0.05$.

\section{Results}

After screening the initial cohort $(N=131)$ for eligibility (Fig. 1), we included 37 patients (age 51.9 $\pm 10.9 ; 25$ men, 12 women), of whom 10 were classified as ME/CFS + (age 
$50.7 \pm 12.3 ; 5$ women) and 27 as ME/CFS- (age 52.3 \pm 10.5 ; 7 women; Table 1 ).

Socio-demographic data, baseline comorbidities, and clinical and inflammatory features of SARS-CoV-2 infection did not differ between groups.

Sleep quality, fatigue, pain, and depressive symptoms were significantly more common and more severe in the ME/ CFS + vs. ME/CFS - group (Fig. 2). Anxiety did not differ between groups.

Subjective cognitive complaints were significantly worse in the ME/CFS + vs. ME/CFS - group $(p<0.001)$, but the neuropsychological battery scores did not differ between groups.

Lung function was within normal range and did not differ between groups, except for Borg dyspnea baseline measure of the 6MWT ( $p=0.014)$, which was significantly higher in the ME/CFS + vs. ME/CFS - group (Fig. 2).

Serum NfL levels were normal in all patients, except one (ME/CFS +, woman, age $54 ; 28.5 \mathrm{pg} / \mathrm{mL}$, normal value $<17.5 \mathrm{pg} / \mathrm{mL}$ ) (Mariotto et al. 2019) and did not differ comparing ME/CFS + and ME/CFS - groups.

\section{Discussion}

We identified a subset of 10 patients (i.e., $27 \%$ of the included patients), who developed ME/CFS-like symptoms of fatigue, sleep disturbances, pain, mood changes, and subjective cognitive complaints persisting over 6 months after SARS-CoV-2 infection recovery. Our figures appear

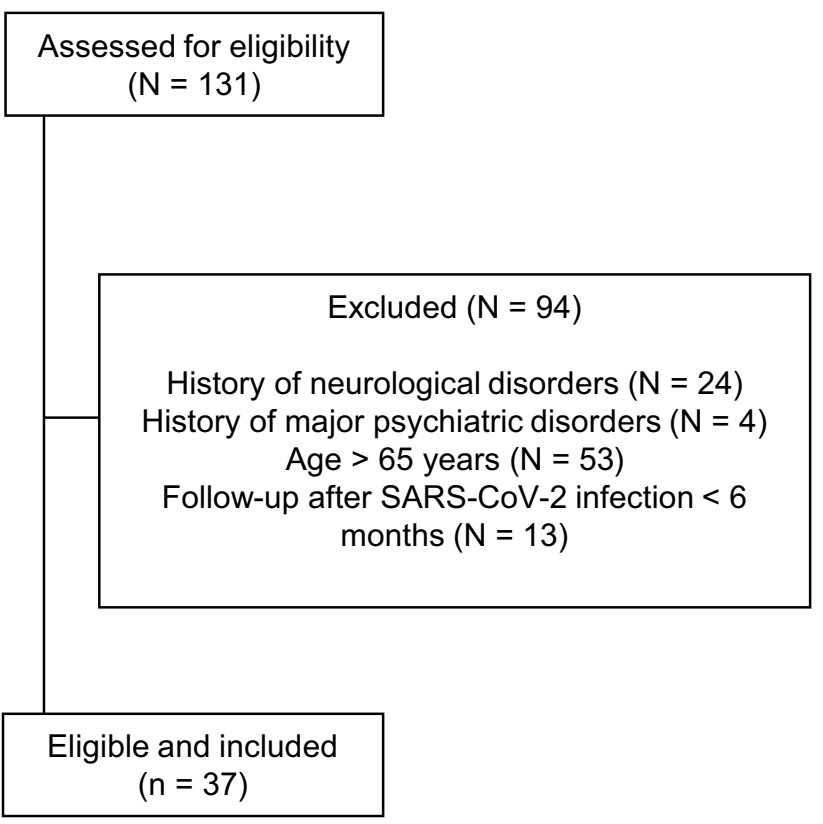

Fig. 1 Flow diagram of the study and reasons for patients' exclusion slightly lower than that previously reported after SARS infection, i.e., ME/CFS-like symptoms in $40 \%$ of survivors 1 year after infection (Lam et al. 2009), but higher than that reported in a recent study aimed to assess psychological morbidities among COVID-19 survivors, i.e., ME/ CFS-like symptoms in $14.2 \%$ of survivors 6 months after infection onset (Simani et al. 2021). These discrepancies across studies might be explained by different ME/CFS criteria and severity of the two coronavirus infections.

In accordance with another study on persistent fatigue after COVID-19 (Ahmed et al. 2020), we did not find significant difference in clinical/inflammatory features of SARS-CoV-2 acute infection comparing ME/CFSlike patients to those without ME/CFS-like symptoms. Although most of the patients were not severely ill, this finding suggests that ME/CFS-like symptoms may be independent from SARS-CoV-2 infection severity.

COVID-19 survivors underwent a multidimensional assessment that showed worse sleep, fatigue pain, depressive symptoms, subjective cognitive complaints, and dyspnea in ME/CFS-like group.

More severe mood alterations were found in ME/ $\mathrm{CFS}+$ than ME/CFS- patients, in line with a recent prospective study on COVID-19 survivors (Rass et al. 2021).

$\mathrm{ME} / \mathrm{CFS}$ + patients reported higher subjective cognitive complaints than the ME/CFS- group, even in the absence of overt cognitive dysfunction. The subjective-objective cognitive mismatch suggests that ME/CFS-like cognitive complaints may fall within the functional cognitive disorder spectrum (Teodoro et al. 2018). Alternatively, the tools we used could be not calibrated to capture subtle cognitive impairment.

Serum NfL levels were normal except in one ME/CFSlike patient, and we found no differences when comparing ME/CFS + vs. ME/CFS - . This finding suggests that axonal damage does not seem to underlie ME/CFS-like symptoms in SARS-CoV-2 survivors and requires confirmation in larger studies.

Lung function was normal and not significantly different in ME/CFS + vs. ME/CFS - groups. Of interest, ME/ CFS-like patients perceived baseline Borg scale dyspnea as more marked than those without ME/CFS-like symptoms. This result appears consistent with a previous study reporting altered dyspnea perception in the absence of objective changes of respiratory distress during a rebreathing test in patients with fibromyalgia and ME/CFS, suggesting a possible functional nature of this symptom (Van den Bergh et al. 2017).

Fatigue, pain, and excessive interoceptive monitoring in ME/CFS may produce a shift from externally directed attention to subjective complaints resulting in perceiving cognitive and motor tasks as extremely effortful (Teodoro et al. 2018). 
Table 1 Socio-demographic and clinical characteristics

\begin{tabular}{|c|c|c|c|c|}
\hline Characteristics & All $(n=37)$ & $\mathrm{ME} / \mathrm{CFS}+(n=10)$ & $\mathrm{ME} / \mathrm{CFS}-(n=27)$ & $P$ value ${ }^{\mathrm{c}}$ \\
\hline \multicolumn{5}{|l|}{ Socio-demographic } \\
\hline Gender (men/women) & $25 / 12$ & $5 / 5$ & $20 / 7$ & 0.17 \\
\hline $\mathrm{Age}^{\mathrm{a}}$ & $51.9 \pm 10.9,54$ & $50.7 \pm 12.3,55.5$ & $52.3 \pm 10.5$ & 0.90 \\
\hline Education $(\mathrm{y})^{\mathrm{a}}$ & $12.9 \pm 3.4,13$ & $14.5 \pm 3.1,15$ & $12.3 \pm 3.3,13$ & 0.07 \\
\hline $\mathrm{BMI}^{\mathrm{a}}$ & $26.8 \pm 4.3,26.4$ & $27.0 \pm 4.4,26.4$ & $26.2 \pm 4.2,26.3$ & 0.90 \\
\hline \multicolumn{5}{|l|}{ Baseline Comorbidities (yes/no) } \\
\hline Hypertension & $9 / 28$ & $4 / 6$ & $5 / 22$ & 0.36 \\
\hline Cardiac disease & $2 / 35$ & $1 / 9$ & $1 / 26$ & 1.00 \\
\hline Diabetes & $1 / 36$ & $0 / 10$ & $1 / 26$ & 1.00 \\
\hline Cancer & $1 / 36$ & $0 / 10$ & $1 / 26$ & 1.00 \\
\hline COPD & $2 / 35$ & $1 / 9$ & $1 / 26$ & 1.00 \\
\hline Kidney disease & $1 / 36$ & $0 / 10$ & $1 / 26$ & 1.00 \\
\hline Liver disease & $0 / 37$ & $0 / 10$ & $0 / 26$ & 1.00 \\
\hline Psychiatric $^{b}$ & $4 / 33$ & $0 / 10$ & $4 / 23$ & 0.49 \\
\hline \multicolumn{5}{|l|}{ SARS-CoV-2 infection-Clinical } \\
\hline Hospitalization (yes/no) & $33 / 4$ & $8 / 2$ & $25 / 2$ & 0.62 \\
\hline Months since infection $^{\mathrm{a}}$ & $6.1 \pm 0.3,6.0$ & $6.1 \pm 0.4,6.0$ & $6.0 \pm 0.1,6.0$ & 0.54 \\
\hline ICU (yes/no) & $8 / 29$ & $3 / 7$ & $5 / 22$ & 0.76 \\
\hline $\mathrm{O}_{2}$ therapy (yes/no) & $22 / 15$ & $4 / 6$ & $18 / 9$ & 0.28 \\
\hline \multicolumn{5}{|l|}{ SARS-CoV-2 infection-Inflammatory } \\
\hline C-reactive protein (mg/L) & $72.4 \pm 52.1,70$ & $72.9 \pm 68.2,83$ & $72.3 \pm 48.0,68$ & 0.84 \\
\hline Procalcitonin $(\mu \mathrm{g} / \mathrm{L})$ & $0.3 \pm 0.4,0.1$ & $0.6 \pm 0.6,0.3$ & $0.3 \pm 0.4,0.1$ & 0.31 \\
\hline White blood cells $(1000 / \mu \mathrm{L})$ & $7.5 \pm 3.6,7.1$ & $8.5 \pm 6.1,6.1$ & $7.2 \pm 2.7,7.1$ & 0.81 \\
\hline Neutrophil granulocytes $(1000 / \mu \mathrm{L})$ & $6.0 \pm 4.0,5.3$ & $7.1 \pm 4.2,5.9$ & $5.7 \pm 4.0,4.7$ & 0.32 \\
\hline Lymphocytes $(1000 / \mu \mathrm{L})$ & $0.9 \pm 0.3,0.9$ & $1.0 \pm 0.4,1.1$ & $0.9 \pm 0.3,0.9$ & 0.27 \\
\hline I nterleukin $6(\mathrm{pg} / \mathrm{mL})$ & $32.5 \pm 23.2,24.0$ & $34.9 \pm 25.1,24.0$ & $31.2 \pm 24.5,25.5$ & 0.80 \\
\hline \multicolumn{5}{|l|}{ Neuropsychiatric symptoms } \\
\hline Sleep disorders (yes/no) & $19 / 18$ & $9 / 1$ & $10 / 17$ & $0.013^{*}$ \\
\hline PSQI score ${ }^{\mathrm{a}}$ & $5.8 \pm 2.8,6.0$ & $7.0 \pm 2.2,6.0$ & $5.3 \pm 2.9,5.0$ & $0.037 *$ \\
\hline \multicolumn{5}{|l|}{ Fatigue } \\
\hline MFI score ${ }^{\mathrm{a}}$ & $42.5 \pm 20.0,36.0$ & $64.4 \pm 17.4,64.0$ & $34.3 \pm 13.7,30.0$ & $<0.001 *$ \\
\hline MFI-FG score ${ }^{a}$ & $9.5 \pm 4.8,8.0$ & $13.6 \pm 4.6,14.0$ & $7.9 \pm 3.9,6.0$ & $0.002 *$ \\
\hline MFI-FF score ${ }^{a}$ & $8.7 \pm 4.7,8.0$ & $13.1 \pm 5.0,12.5$ & $7.0 \pm 3.4,6.0$ & $0.001 *$ \\
\hline MFI-RA score ${ }^{a}$ & $8.7 \pm 4.8,7.0$ & $13.6 \pm 4.7,14.5$ & $6.9 \pm 3.4,6.0$ & $<0.001 *$ \\
\hline MFI-RM score ${ }^{a}$ & $7.5 \pm 3.8,6.0$ & $10.9 \pm 4.1,11.0$ & $6.3 \pm 2.9,5.0$ & $0.001 *$ \\
\hline MFI-FM score ${ }^{\mathrm{a}}$ & $8.0 \pm 4.3,6.0$ & $13.2 \pm 3.5,14.0$ & $6.0 \pm 2.7,5.0$ & $<0.001 *$ \\
\hline Pain (yes/no) & $14 / 23$ & $8 / 2$ & $6 / 21$ & $0.005 *$ \\
\hline NRS score ${ }^{\mathrm{a}}$ & $1.8 \pm 2.5,0.0$ & $4.2 \pm 2.8,5.0$ & $0.9 \pm 1.7,0.0$ & $0.001 *$ \\
\hline Anxiety (yes/no) & $10 / 27$ & $4 / 6$ & $6 / 21$ & 0.51 \\
\hline HADS score (anxiety) ${ }^{\mathrm{a}}$ & $4.6 \pm 3.4,5.0$ & $5.9 \pm 3.5$ & $4.1 \pm 3.5,3.0$ & 0.11 \\
\hline Depression (yes/no) & $6 / 31$ & $4 / 6$ & $2 / 25$ & 0.06 \\
\hline HADS score (depression) ${ }^{\mathrm{a}}$ & $2.9 \pm 3.5,1.0$ & $5.6 \pm 2.8,6.0$ & $1.9 \pm 2.9,1.0$ & $0.002 *$ \\
\hline \multicolumn{5}{|l|}{ Neuropsychological assessment } \\
\hline Subjective complaints (yes/no) & $14 / 23$ & $10 / 0$ & $4 / 23$ & $<0.001 *$ \\
\hline General (MoCA raw score) $)^{\mathrm{a}}$ & $26.0 \pm 2.0,26.0$ & $27.0 \pm 1.6,27.0$ & $25.6 \pm 2.0,26.0$ & 0.05 \\
\hline General (MoCA corrected score) ${ }^{\mathrm{a}}$ & $25.4 \pm 2.4,24.5$ & $26.1 \pm 2.2,26.2$ & $25.1 \pm 2.4,24.5$ & 0.22 \\
\hline Psychomotor speed (SDT score) ${ }^{\mathrm{a}}$ & $46.9 \pm 10.4,46.0$ & $51.3 \pm 8.7,48.5$ & $45.2 \pm 10.7,44.0$ & 0.10 \\
\hline Executive function (Stroop time) $)^{\mathrm{a}}$ & $18.5 \pm 8.3,17.5$ & $16.2 \pm 7.5,16.9$ & $19.4 \pm 8.6,17.8$ & 0.39 \\
\hline Neurofilament light chain $(\mathrm{pg} / \mathrm{mL})$ & $9.5 \pm 4.9,8.5$ & $10.4 \pm 7.7,8.2$ & $9.2 \pm 3.5,8.5$ & 0.89 \\
\hline \multicolumn{5}{|l|}{ Spirometry } \\
\hline FEV1 $(\% \text { predicted })^{\mathrm{a}}$ & $115.9 \pm 15.6,116.0$ & $113.5 \pm 11.2,111.5$ & $116.6 \pm 16.9,119.0$ & 0.36 \\
\hline $\mathrm{FVC}(\% \text { predicted })^{\mathrm{a}}$ & $120.8 \pm 17.0,120.0$ & $114.8 \pm 10.7,117.0$ & $122.7 \pm 18.3,126.0$ & 0.17 \\
\hline $\operatorname{FEV} / \mathrm{FVC}(\%)^{\mathrm{a}}$ & $100.5 \pm 6.7,101.0$ & $103.9 \pm 8.6,107.0$ & $99.4 \pm 5.7,100.0$ & 0.10 \\
\hline $\operatorname{TLC}(\% \text { predicted })^{\mathrm{a}}$ & $102.3 \pm 13.0,103.0$ & $98.3 \pm 12.1,102.5$ & $103.6 \pm 13.3,103.0$ & 0.42 \\
\hline
\end{tabular}


Table 1 (continued)

\begin{tabular}{|c|c|c|c|c|}
\hline Characteristics & All $(n=37)$ & $\mathrm{ME} / \mathrm{CFS}+(n=10)$ & $\mathrm{ME} / \mathrm{CFS}-(n=27)$ & $P$ value $^{\mathrm{c}}$ \\
\hline $\mathrm{DL}_{\mathrm{CO}}(\% \text { predicted })^{\mathrm{a}}$ & $89.1 \pm 13.3,87.0$ & $84.5 \pm 15.0,81.0$ & $90.5 \pm 12.7,87.0$ & 0.23 \\
\hline $\mathrm{K}_{\mathrm{CO}}(\% \text { predicted })^{\mathrm{a}}$ & $95.4 \pm 15.5,93.0$ & $97.6 \pm 21.8,89.5$ & $94.7 \pm 13.4,94.0$ & 0.85 \\
\hline \multicolumn{5}{|l|}{ Arterial blood gas analysis } \\
\hline $\mathrm{pCO}_{2}(\mathrm{~mm} \mathrm{Hg})^{\mathrm{a}}$ & $36.5 \pm 7.2,38.0$ & $38.8 \pm 1.5,39.0$ & $35.8 \pm 8.2,37.0$ & 0.15 \\
\hline $\mathrm{pO}_{2}(\mathrm{~mm} \mathrm{Hg})^{\mathrm{a}}$ & $97.0 \pm 21.7,99.0$ & $99.3 \pm 6.6,100.0$ & $96.2 \pm 24.7,99.0$ & 0.95 \\
\hline \multicolumn{5}{|l|}{ Six-minute walking test } \\
\hline Distance walked $(\mathrm{m})^{\mathrm{a}}$ & $589.4 \pm 66.9,600.0$ & $602.9 \pm 80.2,608.0$ & $584.9 \pm 63.2,576.0$ & 0.27 \\
\hline Distance walked (\% predicted) ${ }^{\mathrm{a}}$ & $103.2 \pm 17.8,100.3$ & $106.0 \pm 18.1,106.5$ & $102.2 \pm 17.9,98.7$ & 0.32 \\
\hline $\mathrm{SatO}_{2}(\text { baseline })^{\mathrm{a}}$ & $97.0 \pm 1.1,97.0$ & $97.1 \pm 0.8,97.0$ & $97.0 \pm 1.1,97.0$ & 0.84 \\
\hline $\mathrm{SatO}_{2}$ (end-effort) ${ }^{\mathrm{a}}$ & $96.6 \pm 1.8,97.0$ & $96.8 \pm 1.7,97.0$ & $96.5 \pm 2.0,96.5$ & 0.77 \\
\hline Borg dyspnea scale (baseline) ${ }^{\mathrm{a}}$ & $0.16 \pm 0.45,0.0$ & $0.50 \pm 0.76,0.3$ & $0.04 \pm 0.20,0.0$ & $0.014 *$ \\
\hline Borg fatigue scale (baseline) ${ }^{a}$ & $0.09 \pm 0.30,0.0$ & $0.25 \pm 0.46,0.0$ & $0.04 \pm 0.20,0.0$ & 0.085 \\
\hline Borg dyspnea scale (end-effort) $)^{\mathrm{a}}$ & $2.16 \pm 1.54,3.0$ & $2.38 \pm 0.92,3.0$ & $2.08 \pm 1.72,2.0$ & 0.55 \\
\hline Borg fatigue scale (end-effort) ${ }^{\mathrm{a}}$ & $1.69 \pm 1.38,1.0$ & $2.13 \pm 1.73,2.0$ & $1.54 \pm 1.25,1.0$ & 0.36 \\
\hline
\end{tabular}

$B M I$ body mass index, $C O P D$ chronic obstructive pulmonary disease, $D L_{C O}$ diffusing capacity of the lungs for carbon monoxide, $F E V_{l}$ forced expiratory volume in the first second, $F V C$ forced vital capacity, $H A D S$ Hospital Anxiety and Depression Scale, $I C U$ intensive care unit, $K_{C O}$ carbon monoxide transfer coefficient, $M E / C F S$ + patients with myalgic encephalomyelitis/chronic fatigue syndrome-like symptoms, $M E /$ $C F S$ - patients without myalgic encephalomyelitis/chronic fatigue syndrome-like symptom, $M F I$ Multidimensional Fatigue Inventory, $M F I-F G$ general fatigue, $M F I-F F$ physical fatigue, MFI-RA reduced activity, MFI-RM reduced motivation, MFI-FM mental fatigue, MoCA Montreal Cognitive Assessment, $\mathrm{NRS}$ numerical rating scale, $\mathrm{pCO}_{2}$ partial pressure of arterial carbon dioxide, $p \mathrm{O}_{2}$ partial pressure of oxygen, $P S Q I$ Pittsburgh Sleep Quality Index, $\mathrm{SatO}_{2}$ oxygen saturation, $S D T$ Symbol Digit Test, $T L C$ total lung capacity

${ }^{\mathrm{a}}$ Mean $\pm \mathrm{SD}$, median

${ }^{\mathrm{b}}$ Mild severity

${ }^{\mathrm{c}}$ Non-parametric Mann-Whitney U test for continuous variables; chi square test for categorical variables

$* P<0.05$ for ME/CFS + vs ME/CFS - comparison

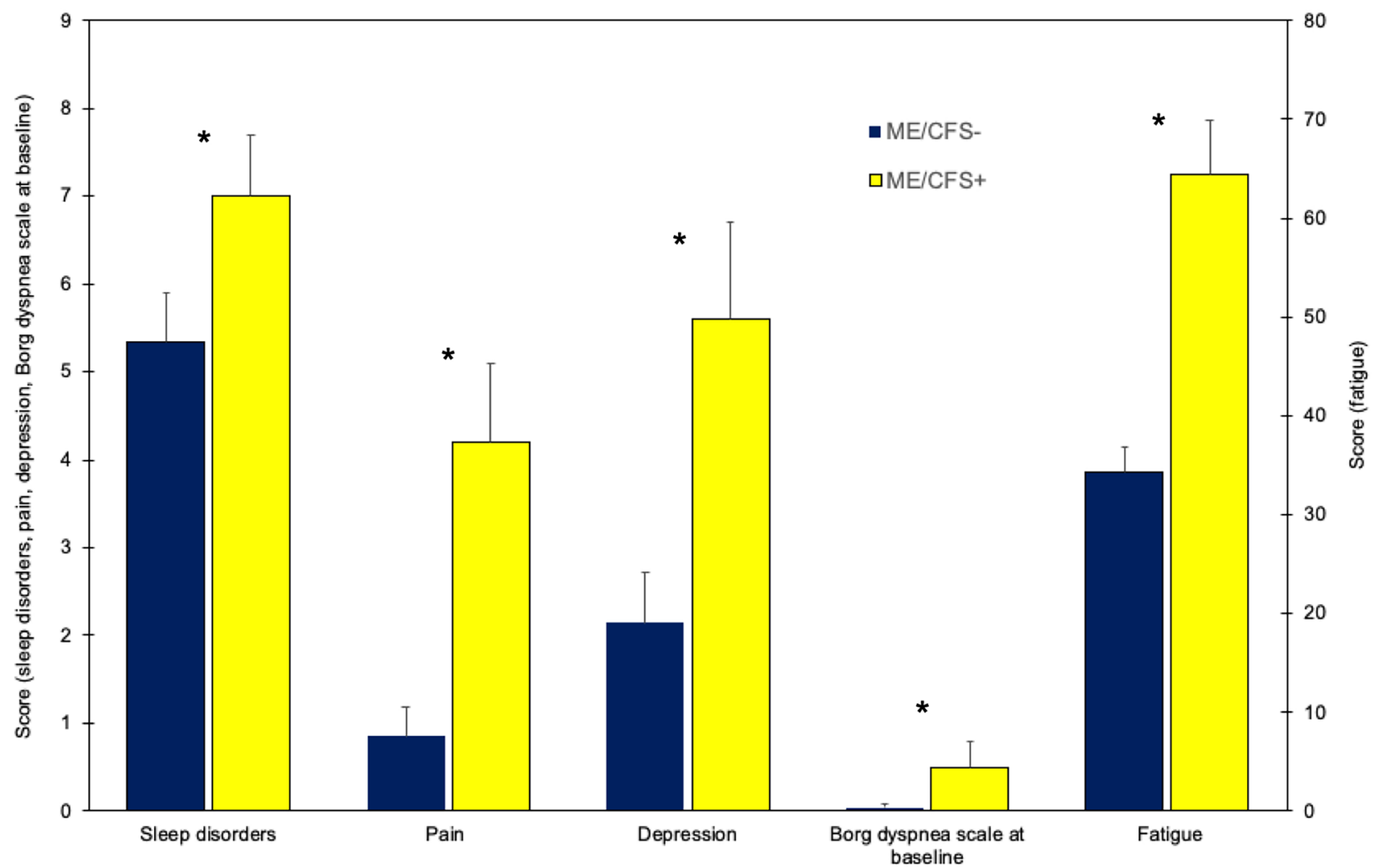

Fig. 2 Sleep disorders, pain, depression, perceived dyspnea (Borg dyspnea scale at baseline), and fatigue in patients who tested positive $(\mathrm{ME} / \mathrm{CFS}+)$ and negative (ME/CFS -) to ME/CFS-like symptoms.
All the measures were significantly worse in $\mathrm{ME} / \mathrm{CFS}$ + than ME/ $\mathrm{CFS}-$ patients $(* p<0.05$ for $\mathrm{ME} / \mathrm{CFS}+\mathrm{vs} \mathrm{ME} / \mathrm{CFS}-$ comparison). Horizontal bars equal 1 S.E.M 
Our results suggest that ME/CFS-like symptoms in patients with COVID-19 may fall under functional neurological syndromes; i.e., a set of disorders characterized by abnormal symptom experiences probably related to an imbalance between somatic bottom-up perception and attentional, affective, and memory top-down processes (Van Den Houte et al. 2018).

Strengths of this study are the 6-month follow-up data and the multidimensional assessment encompassing immunologic, axonal, and lung markers. Limitations include its observational nature, small sample size, the absence of a pre-COVID-19 evaluation that prevented conclusions on the cause-effect relationship, no age- and sex-matched control group of patients without COVID-19 to check for psychological distress caused by the pandemic itself, and no data on inflammatory features at 6-month follow-up. Whether these symptoms might be a consequence of SARS-CoV-2 infection or unmasking of previous latent predisposition is an open question that should be addressed in future studies.

Despite our data should be considered preliminary, they raise concern on a possible future ME/CFS-like pandemic long after SARS-CoV-2 infection recovery, irrespectively of its severity and inflammatory features. Future larger studies with longer follow-ups are needed to explore ME/CFS-like symptom prevalence following SARS-CoV-2 infection.

Author contribution EM: design of the study, acquisition, interpretation of the data, draft and revision of the manuscript for important intellectual content. SM, DG, GD, SB, AF, SZ, DG: acquisition, interpretation of the data, revision of the manuscript for important intellectual content. EC, SF: design of the study, acquisition, interpretation of the data, revision of the manuscript for important intellectual content. ST: design of the study, acquisition, analysis and interpretation of the data, draft and revision of the manuscript for important intellectual content, study supervision. All authors read and approved the final version of the manuscript.

Data availability The data that support the findings of this study are available from the corresponding author upon reasonable request.

\section{Declarations}

Ethics approval Consent to participate was obtained from included patients, and the study was approved by the Ethics Committee of Verona University Hospital (Approval \#2785CESC Verona-Rovigo).

Competing interests The authors declare no competing interests.

\section{References}

Aamodt AH, Høgestøl EA, Popperud TH, Holter JC, Dyrhol-Riise AM, Tonby K, Stiksrud B, Quist-Paulsen E, Berge T, Barratt-Due A,
Aukrust P, Heggelund L, Blennow K, Zetterberg H, Harbo HF (2021) Blood neurofilament light concentration at admittance: a potential prognostic marker in COVID-19. J Neurol 20:1-10. https://doi.org/10.1007/s00415-021-10517-6

Ahmed H, Patel K, Greenwood DC, Halpin S, Lewthwaite P, Salawu A, Eyre L, Breen A, O'Connor R, Jones A, Sivan M (2020) Longterm clinical outcomes in survivors of severe acute respiratory syndrome and Middle East respiratory syndrome coronavirus outbreaks after hospitalisation or ICU admission: a systematic review and meta-analysis. J Rehabil Med 52:jrm00063. https:// doi.org/10.2340/16501977-2694

Carruthers BM, van de Sande MI, De Meirleir KL, Klimas NG, Broderick G, Mitchell T, Staines D, Powles AC, Speight N, Vallings R, Bateman L, Baumgarten-Austrheim B, Bell DS, CarloStella N, Chia J, Darragh A, Jo D, Lewis D, Light AR, MarshallGradisnik S, Mena I, Mikovits JA, Miwa K, Murovska M, Pall ML, Stevens S (2011) Myalgic encephalomyelitis: international consensus criteria. J Intern Med 270:327-338. https://doi.org/10. 1111/j.1365-2796.2011.02428.x

Hviid CVB, Knudsen CS, Parkner T (2020) Reference interval and preanalytical properties of serum neurofilament light chain in Scandinavian adults. Scand J Clin Lab Invest 80:291-295. https:// doi.org/10.1080/00365513.2020.1730434

Johnston S, Brenu EW, Staines D, Marshall-Gradisnik S (2013) The prevalence of chronic fatigue syndrome/myalgic encephalomyelitis: a meta-analysis. Clin Epidemiol 5:105-110. https://doi.org/10. 2147/CLEP.S39876

Lam MH, Wing YK, Yu MW, Leung CM, Ma RC, Kong AP, So WY, Fong SY, Lam SP (2009) Mental morbidities and chronic fatigue in severe acute respiratory syndrome survivors: long-term followup. Arch Intern Med 169:2142-2147. https://doi.org/10.1001/ archinternmed.2009.384

Lee SH, Shin HS, Park HY, Kim JL, Lee JJ, Lee H, Won SD, Han W (2019) Depression as a mediator of chronic fatigue and posttraumatic stress symptoms in Middle East Respiratory Syndrome survivors. Psychiatry Investig 16:59-64. https://doi.org/10.30773/ pi.2018.10.22.3

Mariotto S, Gajofatto A, Zuliani L, Zoccarato M, Gastaldi M, Franciotta D, Cantalupo G, Piardi F, Polo A, Alberti D, Sartori S, Zanusso G, Agrò L, Demurtas R, Sechi G, Sechi E, Monaco S, Ferrari S (2019) Serum and CSF neurofilament light chain levels in antibody-mediated encephalitis. J Neurol 266:643-1648. https://doi.org/10.1007/s00415-019-09306-Z

Mariotto S, Savoldi A, Donadello K, Zanzoni S, Bozzetti S, Carta S, Zivelonghi C, Alberti D, Piraino F, Minuz P, Girelli D, Crisafulli E, Romano S, Marcon D, Marchi G, Gottin L, Polati E, Zanatta P, Monaco S, Tacconelli E, Ferrari S (2020) Nervous system: subclinical target of SARS-CoV-2 infection. J Neurol Neurosurg Psychiatry 91:1010-1012. https://doi.org/10.1136/jnnp-2020-323881

Miller MR, Hankinson J, Brusasco V, Burgos F, Casaburi R, Coates A, Crapo R, Enright P, van der Grinten CP, Gustafsson P, Jensen R, Johnson DC, MacIntyre N, McKay R, Navajas D, Pedersen OF, Pellegrino R, Viegi G, Wanger J, ATS/ERS Task Force, (2005) Standardisation of spirometry. Eur Respir J 26:319-338. https:// doi.org/10.1183/09031936.05.00034805

Rass V, Beer R, Josef Schiefecker A, Kofler M, Lindner A, Mahlknecht P, Heim B, Limmert V, Sahanic S, Pizzini A, Sonnweber T, Tancevski I, Loeffler-Ragg J, Scherfler C, Zamarian L, BellmannWeiler R, Weiss G, Djamshidian A, Kiechl S, Seppi K, Pfausler B, Helbok R (2021) Neurological outcome and quality of life three months after COVID-19: a prospective observational cohort study. Eur J Neurol. https://doi.org/10.1111/ene.14803

Rubin R (2020) As Their Numbers Grow, COVID-19 "Long Haulers" Stump Experts. JAMA. https://doi.org/10.1001/jama.2020.17709

Simani L, Ramezani M, Darazam IA, Sagharichi M, Aalipour MA, Ghorbani F, Pakdaman H (2021) Prevalence and correlates of 
chronic fatigue syndrome and post-traumatic stress disorder after the outbreak of the COVID-19. J Neurovirol 27:154-159. https:// doi.org/10.1007/s13365-021-00949-1

Teodoro T, Edwards MJ, Isaacs JD (2018) A unifying theory for cognitive abnormalities in functional neurological disorders, fibromyalgia and chronic fatigue syndrome: systematic review. J Neurol Neurosurg Psychiatry 89:1308-1319. https://doi.org/10.1136/ jnnp-2017-317823

Townsend L, Dyer AH, Jones K, Dunne J, Mooney A, Gaffney F, O'Connor L, Leavy D, O’Brien K, Dowds J, Sugrue JA, Hopkins D, Martin-Loeches I, Ni Cheallaigh C, Nadarajan P, McLaughlin AM, Bourke NM, Bergin C, O'Farrelly C, Bannan C, Conlon N (2020) Persistent fatigue following SARS-CoV-2 infection is common and independent of severity of initial infection. PLoS ONE 15:e0240784. https://doi.org/10.1371/journal.pone.0240784
Van den Bergh O, Witthöft M, Petersen S, Brown RJ (2017) Symptoms and the body: taking the inferential leap. Neurosci Biobehav Rev 74:185-203. https://doi.org/10.1016/j.neubiorev.2017.01.015

Van Den Houte M, Bogaerts K, Van Diest I, De Bie J, Persoons P, Van Oudenhove L, Van den Bergh O (2018) Perception of induced dyspnea in fibromyalgia and chronic fatigue syndrome. J Psychosom Res 106:49-55. https://doi.org/10.1016/j.jpsychores.2018. 01.007

Publisher's Note Springer Nature remains neutral with regard to jurisdictional claims in published maps and institutional affiliations. 